\title{
How can micelle systems be rebuilt by a heating process?
}

This article was published in the following Dove Press journal:

International Journal of Nanomedicine

9 January 2012

Number of times this article has been viewed

\author{
Miguel Adelino da Silva- \\ Filho' \\ Scheyla Daniela Vieira da \\ Silva Siqueira' \\ Larissa Bandeira Freire' \\ Ivonete Batista de Araújo \\ Káttya Gyselle de Holanda \\ e Silva ${ }^{2}$

\section{Aldo da Cunha Medeiros ${ }^{3}$ Irami Araújo-Filho 3 \\ Anselmo Gomes de \\ Oliveira $^{4}$ \\ Eryvaldo Sócrates Tabosa do Egito ${ }^{2}$} \\ 'Dispersed Systems Laboratory, \\ Federal University of Rio Grande do \\ Norte (UFRN), Natal, Rio Grande \\ do Norte, Brazil; ' 2 Department of \\ Pharmacy, UFRN, Natal, Rio Grande \\ do Norte, Brazil; ; ${ }^{3}$ Department \\ of Experimental Surgery, UFRN, \\ Natal, Rio Grande do Norte, Brazil; \\ ${ }^{4}$ Department of Drugs and Medicines, \\ College of Pharmaceutical Sciences \\ (UNESP), Araraquara, São Paulo, Brazil
}

Correspondence: Eryvaldo Sócrates Tabosa do Egito

Laboratório de Sistemas Dispersos, Rua Praia de Areia Branca, 8948 Natal - RN - Brazil CEP: 59094-450

Tel +55 8494318816

Fax +55843342 98I7

Email socrates@ufrnet.br
Abstract: The aim of this work was to evaluate how an aqueous micellar system containing Amphotericin B (AmB) and sodium deoxycholate (DOC) can be rebuilt after heating treatment. Also, a review of the literature on the physicochemical and biological properties of this new system was conducted. Heated (AmB-DOC-H) and unheated (AmB-DOC) micelles were then diluted at four different concentrations $\left(50 \mathrm{mg} \cdot \mathrm{L}^{-1}, 5 \mathrm{mg} \cdot \mathrm{L}^{-1}, 0.5 \mathrm{mg} \cdot \mathrm{L}^{-1}\right.$, and $\left.0.05 \mathrm{mg} \cdot \mathrm{L}^{-1}\right)$ to perform physicochemical studies and a pharmacotoxicity assay, in which two cell models were used for the in vitro experiments: red blood cells (RBC) from human donors and Candida parapsilosis $(C p)$. While potassium $\left(\mathrm{K}^{+}\right)$and hemoglobin leakage from $\mathrm{RBC}$ were the parameters used to evaluate acute and chronic toxicity, respectively, the efficacy of AmB-DOC and AmB-DOC-H were assessed by $\mathrm{K}^{+}$leakage and cell survival rate from $C p$. The spectral study revealed a slight change in the AmB-DOC aggregate peak from $327 \mathrm{~nm}$ to $323 \mathrm{~nm}$, which is the peak for AmB-DOC-H. Although AmB-DOC and AmB-DOC-H exhibited different behavior for hemoglobin leakage, AmB-DOC produced higher leakage than AmB-DOC-H at high concentrations (from $5 \mathrm{mg} \cdot \mathrm{L}^{-1}$ ). For $\mathrm{K}^{+}$leakage, both AmB-DOC and AmB-DOC-H showed a similar profile for both cell models, RBC and $C p(P<0.05)$. AmBDOC-H and AmB-DOC also revealed a similar profile of activity against $C p$ with an equivalent survival rate. In short, AmB-DOC-H showed much less toxicity than AmB-DOC, but remained as active as AmB-DOC against fungal cells. The results highlight the importance of this new procedure as a simple, inexpensive, and safe way to produce a new kind of micelle system for the treatment of systemic fungal infections.

Keywords: micelles, nanotechnology, pre-heated amphotericin B, super-aggregates

\section{Introduction}

Amphotericin B (AmB) is a water-insoluble compound widely used to effectively treat systemic fungal infections. ${ }^{1}$ However, its utilization as an antifungal agent has been limited by high acute and chronic toxicity, characterized by chills, fever, nausea, vomiting, and nephrotoxicity. ${ }^{2-4}$

Due to the amphiphilic nature of the molecule and its poor solubility in water, $\mathrm{AmB}$ is able to self-associate in aqueous media and form supramolecular aggregates. However, in aqueous media, monomers and soluble and insoluble aggregates of AmB coexist, the latter being responsible for the toxic effects. ${ }^{5-8}$ When AmB in solution is below the minimal concentration for self-association, toxicity against fungi is still observed, while the human toxic effect decreases. ${ }^{2,3}$

In the current model of AmB selective toxicity, soluble monomeric AmB is able to form ion channels in ergosterol-rich fungal membranes, but a soluble self-associated 
oligomer damages sterol-free and cholesterol-containing membranes. ${ }^{9,10}$ Also, the oligomers seem to be susceptible to oxidation, which may enhance their toxicity. ${ }^{11}$ Therefore, the way in which AmB is cleared from the body plays an important role in its toxicity and activity. The widely-accepted mechanism of action of AmB described above is true for micelle systems; the AmB molecules bind to a surfactant with a high degree of freedom and can easily leave the surfactant molecule and self-associate.

The micellar deoxycholate system of AmB (AmB-DOC), a well-known commercial antifungal product $\left(\right.$ Fungizon $\left.^{\circledR}\right)$, has been used in clinical practice for over 45 years for the treatment of several systemic fungal infections. ${ }^{2,12}$ Unfortunately, AmB-DOC shows a concentration-dependent profile of toxicity and its selectivity depends on its aggregation state. Clinically, nephrotoxicity is frequently observed. ${ }^{13}$

In an attempt to reduce the AmB-DOC toxicity, some lipid formulations such as liposomes and other AmB lipidassociated forms were developed. ${ }^{14-18}$ These systems are able to immobilize the AmB monomers, preventing selfaggregation. They slowly release monomers to the media, and, therefore, are active only against fungal cells. Although these formulations exhibit a far superior therapeutic index compared to AmB-DOC, they are costly and their clinical use is inaccessible for many countries where fungal-related diseases are rampant. In fact, only AmB-DOC is widely used in some developing countries, and after the 1990s, when Fungizon ${ }^{\circledR}$ was no longer patent-protected, this product started to be manufactured for several brands as a generic product.

The literature indicates that, when subjected to controlled heat, AmB-DOC forms a new type of aggregate, which is less susceptible to oxidative degradation and less selective for the cholesterol in the mammalian cell membrane, thus making it less toxic. ${ }^{19-25}$ The heating of AmB-DOC solutions generates "super-aggregates," in which the toxicity is significantly reduced while keeping their antifungal activity. However, a recent study conducted in our research group revealed that AmB micellar systems made by different companies present different profiles of activity and toxicity, probably due to the variation in the formation of the structure of the drug-surfactant micelle system as a result of changes in the manufacturing process or because of the quality of the raw materials used (our unpublished results).

The aim of this work was to evaluate the relationship between controlled heat treatment, the absorption spectra, and the pharmacotoxicity of a Brazilian brand of AmB-DOC in aqueous micellar solutions before and after heat treatment
(AmB-DOC-H). In this way, a new process to rebuild a pre-formed micelle system can be achieved.

\section{Materials and methods Preparation of the AmB-DOC and AmB-DOC-H samples}

The stock solution of AmB-DOC (Cristália, Itapira, Brazil) at $5 \times 10^{-3} \mathrm{M}\left(5,000 \mathrm{mg} \cdot \mathrm{L}^{-1}\right)$ was prepared by adding $10 \mathrm{~mL}$ of water for injection into the vial and submitting it to vortex shaking until complete dissolution of the lyophilized powder. Each vial of AmB-DOC contained $50 \mathrm{mg}$ of AmB, approximately $41 \mathrm{mg}$ of sodium deoxycholate, and phosphate buffer, pH 7.4.

Samples of AmB-DOC were prepared with different concentrations by successive dilutions 1:10 of AmB-DOC stock solution in order to obtain the concentrations of $5 \times 10^{-5} \mathrm{M}\left(50 \mathrm{mg} \cdot \mathrm{L}^{-1}\right), 5 \times 10^{-6} \mathrm{M}\left(5 \mathrm{mg} \cdot \mathrm{L}^{-1}\right), 5 \times 10^{-7} \mathrm{M}$ $\left(0.5 \mathrm{mg} \cdot \mathrm{L}^{-1}\right)$, and $5 \times 10^{-8} \mathrm{M}\left(0.05 \mathrm{mg} \cdot \mathrm{L}^{-1}\right)$. The heated AmB (AmB-DOC-H) was prepared by treatment with controlled heat of the AmB-DOC stock solution at $70^{\circ} \mathrm{C}$ for 20 minutes in a thermostatic bath without stirring. The temperature and the time exposition of the bath were checked by a built-in thermometer (Incoterm, Brazil) and chronometer (Model Labor, Hanhart, Germany), respectively. Then, following the same procedure as that for AmB-DOC, dilutions were prepared to perform a similar study of concentration samples.

\section{Spectral study}

Scanning spectra of both AmB-DOC and AmB-DOC-H at the four previously mentioned concentrations were taken by using a UV-VIS Spectrophotometer (Biochrom Libra S32, Cambridge, UK). The optical path of the quartz cuvettes used was $0.1 \mathrm{~cm}$ for the concentration of $5 \times 10^{-5} \mathrm{M}\left(50 \mathrm{mg} \cdot \mathrm{L}^{-1}\right)$; a $1 \mathrm{~cm}$ path for $5 \times 10^{-6} \mathrm{M}\left(5 \mathrm{mg} \cdot \mathrm{L}^{-1}\right)$, and a $10 \mathrm{~cm}$ path for the concentrations of $5 \times 10^{-7} \mathrm{M}\left(0.5 \mathrm{mg} \cdot \mathrm{L}^{-1}\right)$ and $5 \times 10^{-8} \mathrm{M}$ $\left(0.05 \mathrm{mg} \cdot \mathrm{L}^{-1}\right)$. These paths were chosen to obtain spectra with absorbance values less than 0.8 . Their molar extinction coefficients $(\varepsilon)$ were calculated using the Beer-Lambert equation. All spectra were recorded at $25^{\circ} \mathrm{C} \pm 0.1^{\circ} \mathrm{C}$ with a $300-450$ nm range. ${ }^{6}$

\section{Preparation of red blood cell (RBC) suspension}

This study was previously approved by the Ethical Research Committee of the Federal University of Rio Grande do Norte, protocol number 002/2009. In order to minimize sources of variability, one healthy female adult donor, who gave her writ- 
ten informed consent before participating in the study, provided all normal human RBCs for the in vitro experiments. Five milliliters of venous blood was collected in sterile EDTA $(1 \mathrm{mg} / \mathrm{mL}$, ethylene-diamine-tetraacetate at $10 \%$ $(\mathrm{w} / \mathrm{v})$, Labtest, Lagoa Santa, Brazil) syringes and promptly centrifuged (Refrigerated centrifuge, ALC, Model PK121R, Milan, Italy) in tubes at $1,100 \mathrm{~g}$ for 10 minutes at $4^{\circ} \mathrm{C}$. Plasma was carefully aspirated and the exposed buffy coat was removed and discarded. The RBCs were washed three times by centrifugation $\left(1,100 \mathrm{~g}\right.$ for 5 minutes at $\left.4{ }^{\circ} \mathrm{C}\right)$ and suspended in 5 volumes of normal saline $[\mathrm{NaCl}$ at $0.9 \%(\mathrm{w} / \mathrm{v})$, B. Braun, São Paulo, Brazil]. They were then resuspended in $4 \mathrm{~mL}$ of saline, counted in a Neubauer ${ }^{\mathrm{TM}}$ chamber (Splabor, São Paulo, Brazil), and resuspended again until the desired concentration $\left(5 \times 10^{7}\right.$ cells $\left.\cdot \mathrm{mL}^{-1}\right)$ was achieved. The cells were used on the day of collection. ${ }^{17}$

\section{Preparation of Candida parapsilosis suspension}

A strain of Candida parapsilosis (Cp) ATCC (22019) was maintained on Sabouraud- Dextrose-Chloramphenicol agar (SDC, MicroMed, São Paulo, Brazil) at room temperature and passaged monthly. Before experiments, an inoculum from the culture was transferred to a SDC agar scope and incubated at $37^{\circ} \mathrm{C}$ for $16-18$ hours. The fungal cells were then washed three times with normal saline, resuspended, counted in the central reticule of a Neubauer ${ }^{\mathrm{TM}}$ chamber, and resuspended again to obtain the desired cell concentration $\left(5 \times 10^{7} \mathrm{cfu} \cdot \mathrm{mL}^{-1}\right) \cdot{ }^{17}$

\section{Evaluation of the AmB-DOC and AmB-DOC-H toxicity}

Because the AmB molecule binds to human cholesterol of the plasmatic membranes and also to ergosterol in fungal membranes forming hydrophilic pores ${ }^{1}$, analysis of the toxicity and efficacy of the AmB-DOC and AmB-DOC-H formulations was performed using two cell models, RBCs and $C p$. The potassium ions $\left(\mathrm{K}^{+}\right)$inside the cells can be released by any disturbance in the cell membrane due to the pore formation process. In RBCs the generated pore enables the output of $\mathrm{K}^{+}$, but not the release of hemoglobin, which occurs only after the total destruction of the membrane barrier. The phenomenon of $\mathrm{K}^{+}$release from model cells has been referred to as a chronic toxicity by some authors. ${ }^{6,17}$ On the other hand, a strong toxicity level, which induces hemoglobin leakage, for $\mathrm{RBCs}$, or cell death for $C p$, has been classified as acute toxicity. ${ }^{6,17}$
Four milliliter samples of RBCs $\left(5 \times 10^{7}\right.$ cells $\left.\cdot \mathrm{mL}^{-1}\right)$ were incubated for 1 hour at $37^{\circ} \mathrm{C}$ with the vehicle control or with different concentrations $(50,5,0.5$, and $0.05 \mathrm{mg} \cdot \mathrm{L}^{-1}$ ) of both AmB-DOC and AmB-DOC-H. The $\mathrm{RBCs}$ were then centrifuged for 5 minutes at $1,100 \mathrm{~g}$ and washed three times with normal saline. The pellet of RBC was lysed by adding $4 \mathrm{~mL}$ of distilled water and then stirred and centrifuged (1,100 g for 10 minutes) in order to remove membranes. The $\mathrm{K}^{+}$content of the supernatant was determined using a Flame Photometer 7000 (910M Analyser, São Paulo, Brazil) calibrated with $\mathrm{K}^{+}$reference at $5 \mathrm{mEq} \cdot \mathrm{L}^{-1}$. Hemoglobin was estimated from its absorption at $540 \mathrm{~nm}$ recorded on a UV-VIS Spectrophotometer (Biochrom Libra S32). The total $\mathrm{K}^{+}$and hemoglobin content was measured for the control RBC tubes. Release was calculated as the difference between control and treated cells and expressed as a percentage of the total hemoglobin or $\mathrm{K}^{+}$content. At least three different experiments were performed with each formulation and each experimental point was performed in triplicate. ${ }^{26}$

\section{Evaluation of the AmB-DOC and AmB-DOC-H efficacy}

Two milliliter samples of fungal suspension containing $5 \times 10^{7} \mathrm{cfu} \cdot \mathrm{mL}^{-1}$ were incubated for 1 hour at $37^{\circ} \mathrm{C}$ with both AmB-DOC and AmB-DOC-H at the concentrations of $50,5,0.5$, and $0.05 \mathrm{mg} \cdot \mathrm{L}^{-1}$. Cells were centrifuged for 10 minutes at 2,200 $\mathrm{g}$ and washed three times in normal saline, and $2 \mathrm{~mL}$ of purified water was added to the pellet of fungal cells. An aliquot of this pellet was lysed by heating for 5 minutes at $100^{\circ} \mathrm{C}$ and centrifuged to remove membranes, and free $\mathrm{K}^{+}$was measured. The $\mathrm{K}^{+}$leakage was calculated similarly to the calculation of the RBCs. For the cell viability evaluation, $100 \mu \mathrm{L}$ aliquots of appropriate dilution of the fungal pellet were seeded, in duplicate, onto agar plates and incubated at $37^{\circ} \mathrm{C}$. The number of colonyforming units (CFU) was counted at 24 and 48 hours and expressed as a percentage of those obtained from a control inoculum incubated without AmB-DOC or AmB-DOC-H. Three different experiments were performed with each formulation and each experimental point was performed in duplicate. ${ }^{26}$

\section{Statistical analyses}

The statistical results were performed with ANOVA and $t$ test to analyze the variation response in the same group and in different groups, respectively, using Prim 4 for Windows 4.02 (GraphPad Software, San Diego, CA). 


\section{Results}

\section{Aggregation state behavior of AmB-DOC and AmB-DOC-H}

The AmB-DOC-H showed spectra similar to that of AmBDOC, which were concentration-dependent (Figures 1 and 2). At a low concentration, such as $5.10^{-8} \mathrm{M}$, the AmB presented a spectrum with three well-defined absorption bands, with $\lambda_{\text {max }}$ at 363,385 , and $408 \mathrm{~nm}$, and a shoulder around $347 \mathrm{~nm}$, which is similar to that obtained with organic solvents such as methanol, for the monomeric AmB. However, by increasing the concentration of $\mathrm{AmB}$, a new band appeared at $\lambda_{\text {max }}$ $327 \mathrm{~nm}$ at the expense of the bands recorded in the longer wavelengths (363, 385, and $408 \mathrm{~nm}$ ), especially the one characteristic of the monomeric AmB species at $408 \mathrm{~nm}$, which is responsible for the biological activity against fungal cells ${ }^{10}$. The band at $327 \mathrm{~nm}$ was reported in the literature as indicative of the presence of self-associated species of AmB. This band was also sensitive to $\mathrm{AmB}$ concentration, being very well defined at high concentrations as in the range of $10 \times 10^{-7}$ to $10^{-5} \mathrm{M}^{10}$

Concerning the AmB-DOC-H spectra, the band at $327 \mathrm{~nm}$ was slightly blue-shifted to $323 \mathrm{~nm}$ after heating. Several authors have stated that this band is characteristic of the formation of AmB super-aggregates. ${ }^{20}$ It is important to note that the variation in the molar extinction coefficient of $\mathrm{AmB}$ at $\lambda_{\max } 323 \mathrm{~nm}$ and the peaks at 363,385 , and $408 \mathrm{~nm}$ are related to variation in the equilibrium between the monomeric and aggregated species of $\mathrm{AmB}$, which displays an isosbestic point at $347 \mathrm{~nm}$. See scheme.

$$
\mathrm{AmB}_{\mathrm{m}} \stackrel{\mathrm{K}}{\rightleftarrows} \mathrm{AmB}_{\mathrm{sa}} \quad \text { (scheme 1) }
$$

where the underlined letters $m$ and sa refer to the monomeric and self-associated $\mathrm{AmB}$, respectively, while $\mathrm{K}$ is the equilibrium constant between the AmB species.

This equilibrium shows that at low concentrations the monomeric species of $\mathrm{AmB}_{\mathrm{m}}$ prevails, but when the concentration is increased the equilibrium is shifted favoring the predominance of the self-aggregated $\mathrm{AmB}_{\mathrm{sa}}$.

The most important difference among the AmB-DOC and AmB-DOC-H spectra occurred at the concentration of $5 \times 10^{-7} \mathrm{M}$, in which the band at $323 \mathrm{~nm}$ presented a higher molar extinction coefficient $(\varepsilon=71,000)$ compared to the one assigned for AmB-DOC $(\varepsilon=48,666)$. It should be emphasized that the spectra at $5 \times 10^{-8} \mathrm{M}$ were given to illustrate the tendency of concentration dependence. In fact, they should not be considered on a quantitative basis because of the weakness of the signals at the low wavelength region (maximum absorbance at $408 \mathrm{~nm}$ was 0.071 for AmB-DOC).

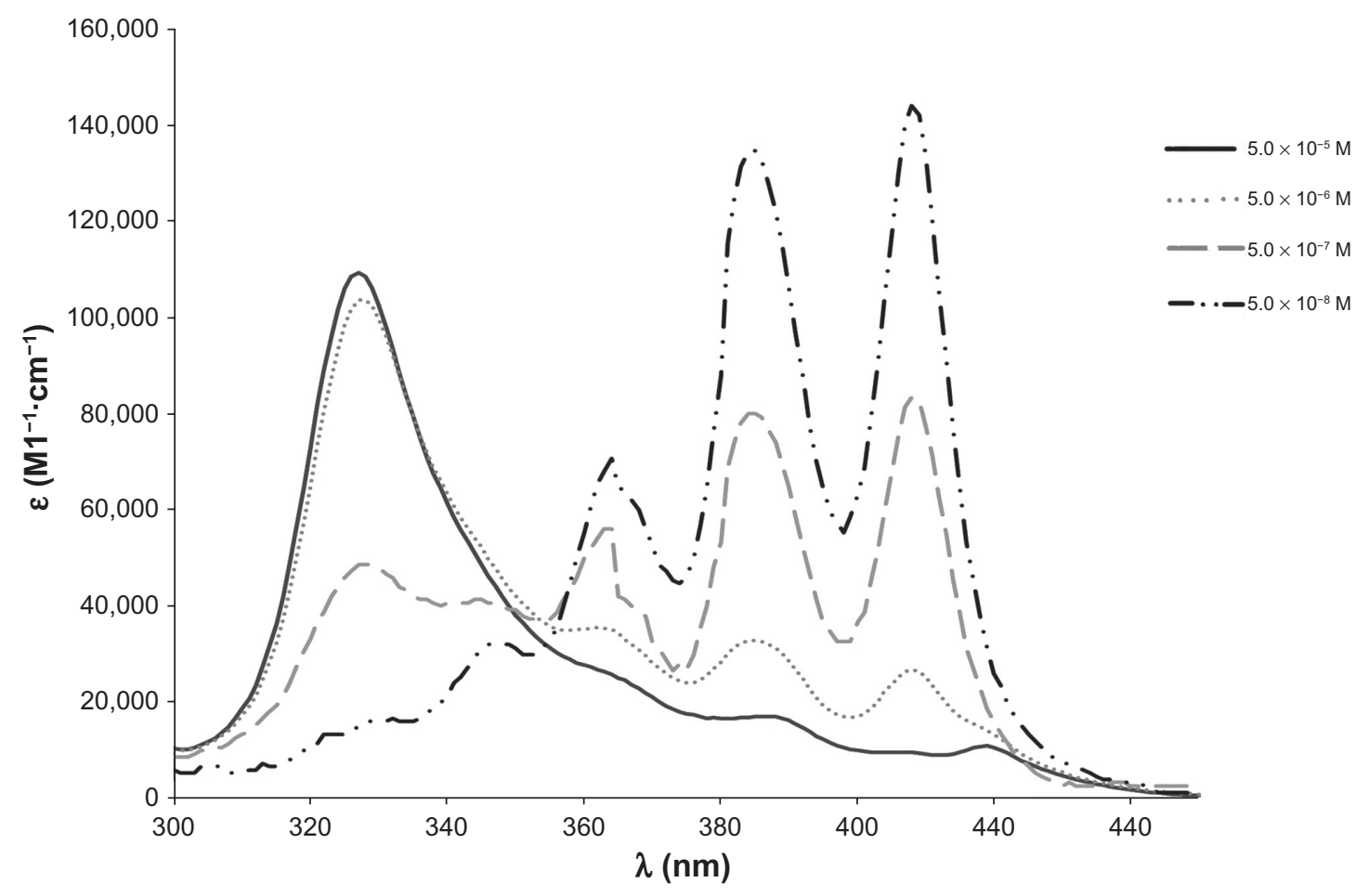

Figure I Concentration-induced changes in the AmB-DOC spectra at $25^{\circ} \mathrm{C}$ at $5 \times 10^{-5} \mathrm{M}$. Abbreviation: AmB-DOC, amphotericin B with sodium deoxycholate. 


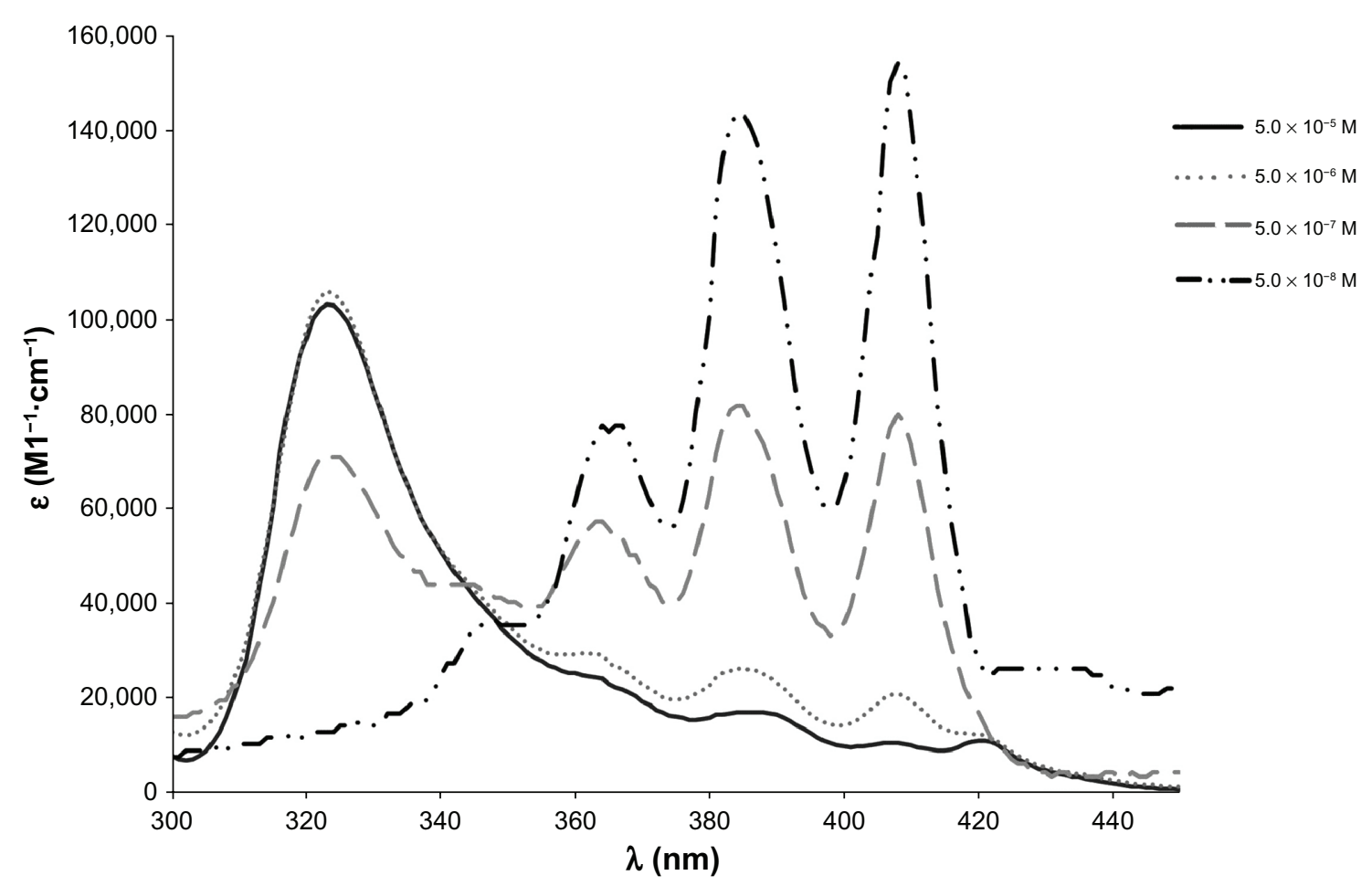

Figure 2 Concentration-induced changes in the AmB-DOC-H spectra at $25^{\circ} \mathrm{C}$ at $5 \times 10^{-5} \mathrm{M}$.

Abbreviation: AmB-DOC, amphotericin B with sodium deoxycholate.

The data for Figures 1 and 2 show that the recorded spectral differences can be attributed to the different interactions of $\mathrm{AmB}$ with the surfactant aggregates provoked by controlled heating, and such changes remained over the whole range of concentrations. Therefore, the heated micelle process was able to produce AmB super-aggregated micelle systems that remained stable after the dilution process. Also, the physicochemical stability of such super-aggregated forms was demonstrated by the absence of changes on the spectra measured on different days over three months (results not shown).

Three decades ago, Ernst et $\mathrm{al}^{28}$ showed the thermally induced increase in aggregate size of Fungizon ${ }^{\circledR}$ aqueous solution at $10^{-5} \mathrm{M}$. This phenomenon was more evident at $60^{\circ} \mathrm{C}$ and increased rapidly at higher temperatures. In an aqueous solution at $70^{\circ} \mathrm{C}$, the apparent mass of the aggregates was 500 -fold larger than the one at $20^{\circ} \mathrm{C} .{ }^{20}$ Therefore, the term super-aggregate used for this new species of $\mathrm{AmB}$ is completely justified. ${ }^{20}$

\section{In vitro toxicity assay for AmB-DOC and AmB-DOC-H}

The toxicity of both AmB-DOC and AmB-DOC-H against RBCs was quite interesting. For $\mathrm{K}^{+}$leakage, both profiles were identical (Figure 3). No significant $\mathrm{K}^{+}$leakage was observed at $0.05 \mathrm{mg} \cdot \mathrm{L}^{-1}\left(5 \times 10^{-8} \mathrm{M}\right)$. At higher concentrations, this completely changes. At $0.5 \mathrm{mg} \cdot \mathrm{L}^{-1}$ $\left(5 \times 10^{-7} \mathrm{M}\right)$, both products induced strong permeability of the cell membrane as reflected by a $\mathrm{K}^{+}$release of around $50 \%$ $(59.59 \% \pm 0.41 \%$ and $50.12 \% \pm 0.9 \%$ for AmB-DOC and AmB-DOC-H, respectively). At the highest concentrations of $5 \mathrm{mg} \cdot \mathrm{L}^{-1}\left(5 \times 10^{-6} \mathrm{M}\right)$ and $50 \mathrm{mg} \cdot \mathrm{L}^{-1}\left(5 \times 10^{-5} \mathrm{M}\right)$, where the aggregate species of $\mathrm{AmB}$ predominates, both systems presented a total release of $\mathrm{K}^{+}$.

Both AmB-DOC and AmB-DOC-H produced no significant hemoglobin leakage below $0.5 \mathrm{mg} \cdot \mathrm{L}^{-1}$ (Figure 4). While the AmB-DOC-H showed no toxic effect on the whole range of the tested concentrations $\left(0.05\right.$ to $50 \mathrm{mg} \cdot \mathrm{L}^{-1}$, $P<0.001)$, the AmB-DOC revealed a sharp increase in the toxicity from $0.5 \mathrm{mg} \cdot \mathrm{L}^{-1}$, reaching to fully lysed cells from $5 \mathrm{mg} \cdot \mathrm{L}^{-1}$ with total hemoglobin leakage to external media.

\section{In vitro activity assay for AmB-DOC and AmB-DOC-H}

The in vitro activity against $C p$ showed that both AmBDOC and AmB-DOC-H were very effective (Figure 5) and that the profile of $\mathrm{K}^{+}$release was quite similar (Figure 3). In fact, a significant $\mathrm{K}^{+}$leakage was found at the lowest concentration $\left(0.05 \mathrm{mg} \cdot \mathrm{L}^{-1}\right)$, reaching around $85 \%$ at the highest concentration. In this assay AmB-DOC-H was 


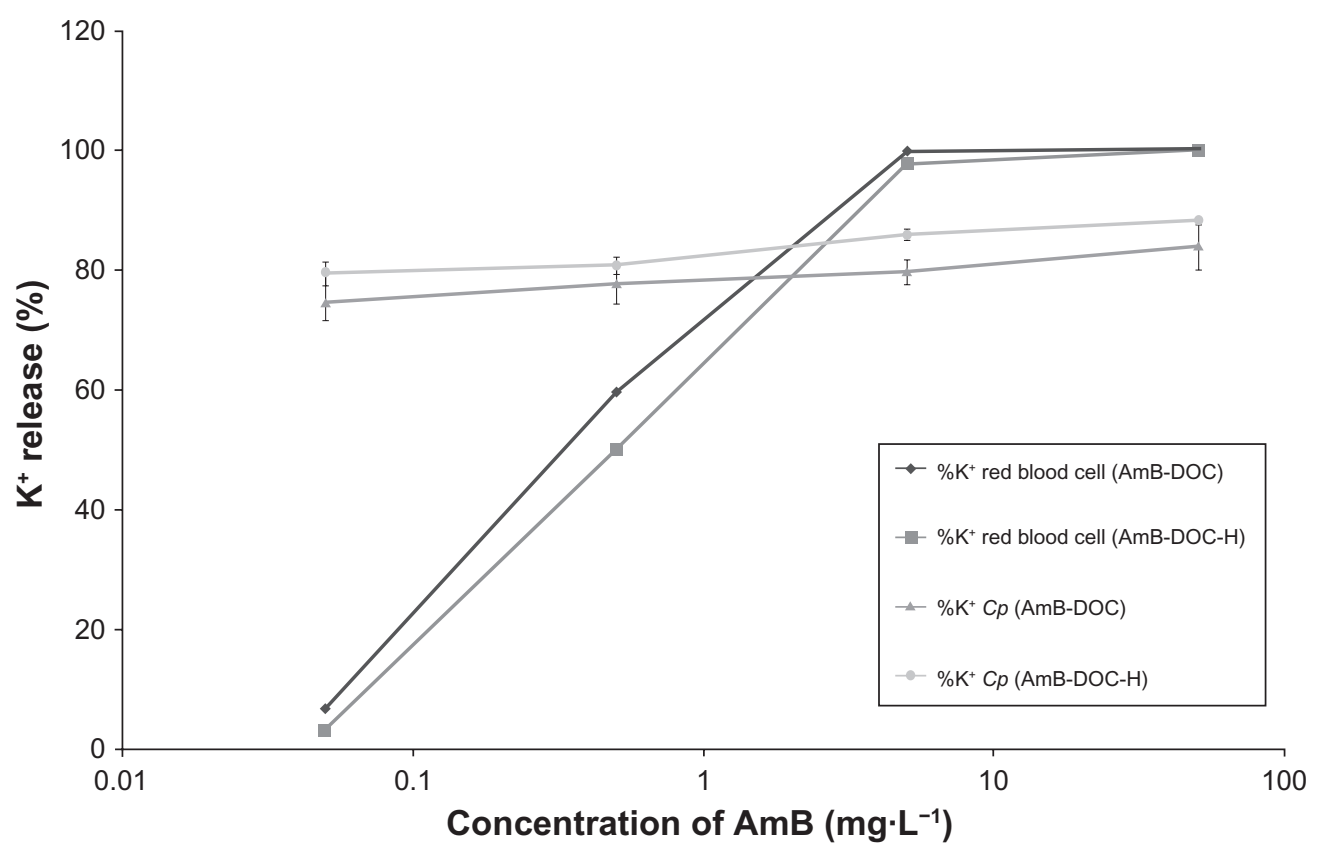

Figure 3 In vitro release of potassium from human RBCs and Candida parapsilosis induced by AmB-DOC and AmB-DOC-H.

Note: Each point on the figure is the mean $( \pm S D)$ of three determinations.

Abbreviations: AmB-DOC, amphotericin B with sodium deoxycholate; AmB-DOC-H, amphotericin B with sodium deoxycholate, heated; RBC, red blood cells.

slightly more active than AmB-DOC over the whole range of concentration. For example, at $5 \mathrm{mg} \cdot \mathrm{L}^{-1} \mathrm{AmB}-\mathrm{DOC}-\mathrm{H}$ presented a $\mathrm{K}^{+}$release of $85.92 \% \pm 0.85 \%$ while $\mathrm{AmB}-\mathrm{DOC}$ showed a release of $79.75 \% \pm 1.96 \%$. At $50 \mathrm{mg} \cdot \mathrm{L}^{-1}$ these values were $88.38 \pm 0.70 \%$ and $83.98 \pm 3.86 \%$, respectively. However, such values were not statistically significant.
These data are in agreement with the results of the cell survival rate, which indicated for both AmB_DOC and AmB-DOC-H a significant decrease in the rate of survival cell at concentrations up to $0.5 \mathrm{mg} \cdot \mathrm{L}^{-1}$. At this concentration range, the AmB-DOC-H showed higher antifungal activity than the unheated AmB. For example,

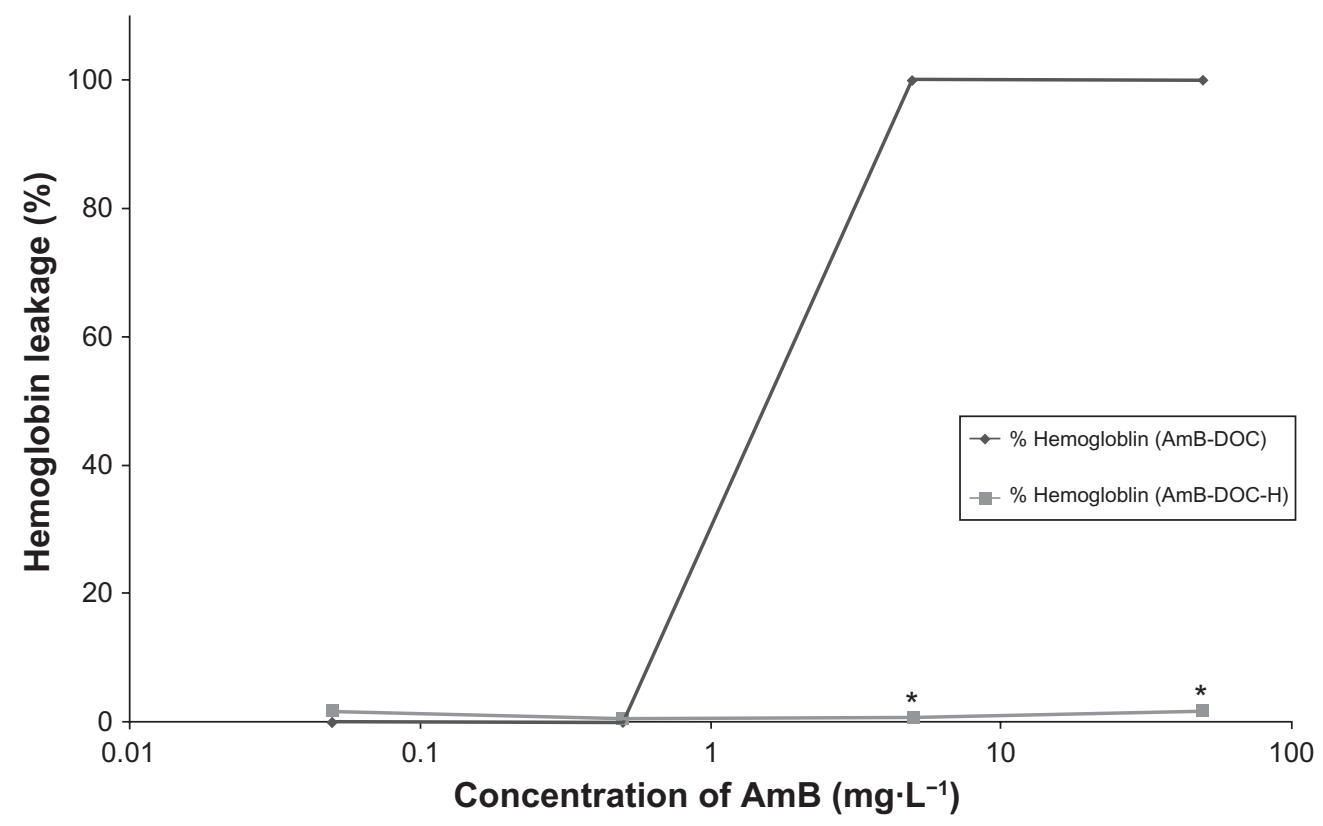

Figure 4 In vitro release of hemoglobin from human RBCs induced by AmB-DOC and AmB-DOC-H.

Notes: Each point on the figure is the mean $( \pm S D)$ of three determinations. *Significant difference between both products $(P<0.00 \mathrm{I})$

Abbreviations: AmB-DOC, amphotericin B with sodium deoxycholate; AmB-DOC-H, amphotericin B with sodium deoxycholate, heated; RBC, red blood cells. 


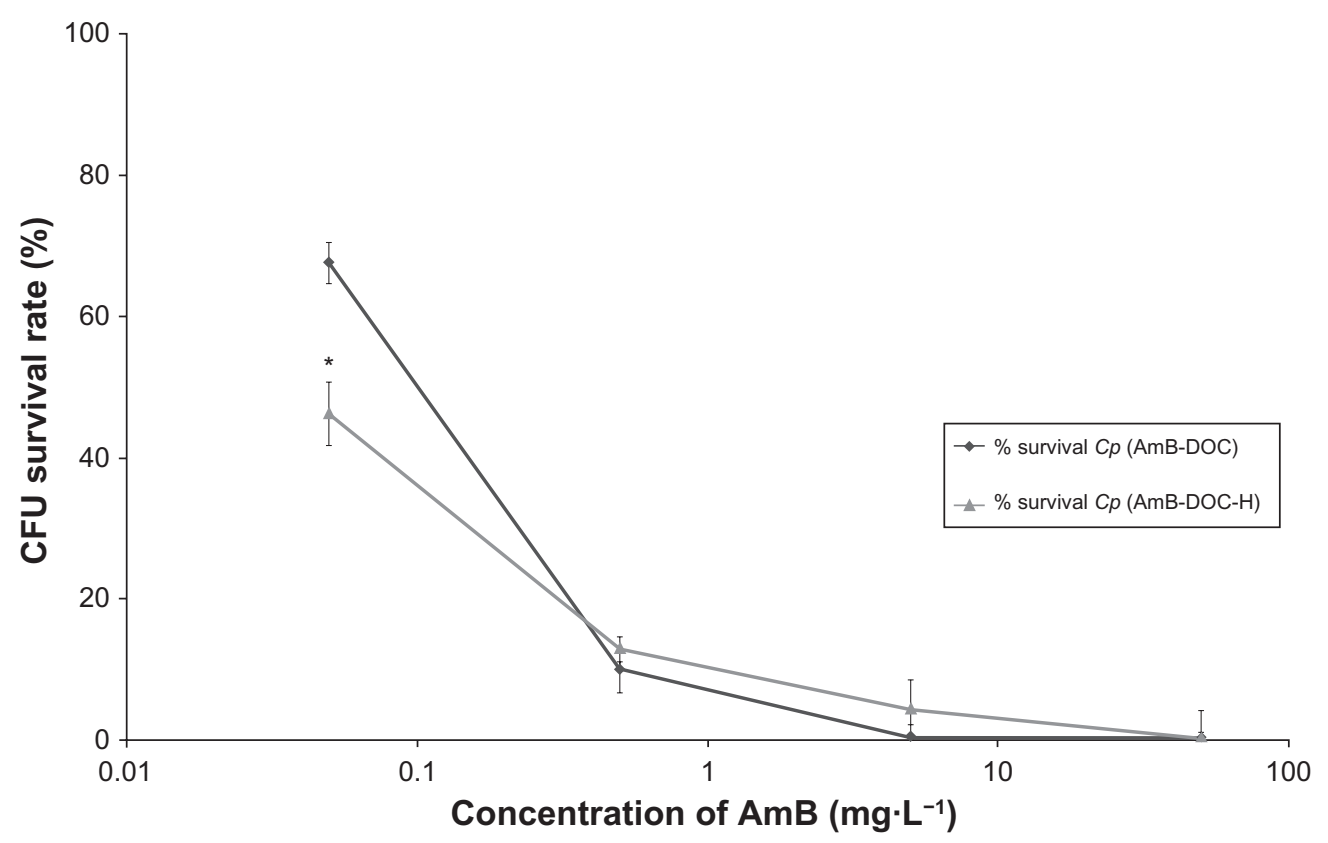

Figure 5 In vitro antifungal activity of AmB-DOC and AmB-DOC-H on C. parapsilosis.

Notes: Each point on the figure is the mean $( \pm S D)$ of three determinations. *Significant difference between both products $(P<0.00 \mathrm{I})$.

Abbreviations: AmB-DOC, amphotericin B with sodium deoxycholate; AmB-DOC-H, amphotericin B with sodium deoxycholate, heated; CFU, colony-forming unit; RBC, red blood cells.

at the concentration of $0.05 \mathrm{mg} \cdot \mathrm{L}^{-1} \mathrm{AmB}-\mathrm{DOC}-\mathrm{H}$ was able to kill $53.72 \% \pm 5.17 \%$ whereas AmB-DOC killed only $32.31 \% \pm 1.40 \%$. Considering the standard deviation, in the range of concentrations from $0.5 \mathrm{mg} \cdot \mathrm{L}^{-1}$ to $50 \mathrm{mg} \cdot \mathrm{L}^{-1}$, the CFU survival rate profile was similar for both preparations. Finally, at $50 \mathrm{mg} \cdot \mathrm{L}^{-1}$ both products were able to kill the totality of the fungal cells.

\section{Discussion}

Micelle systems have been used as nanotechnologic carriers for more than 60 years. $^{2}$ Particularly for AmB, this system has been used since 1957 when Bartner et al studied the incorporation of $\mathrm{AmB}$ molecules into a micellar sodium deoxycholate (DOC), in a molar ratio of approximately 1:2 AmB:DOC, respectively. ${ }^{27}$

The idea that a micellar complex could change its physicochemical property by heating remained completely undiscovered since the first works of Ernst et al. ${ }^{28}$ Almost twenty years later, Gaboriau et $\mathrm{al}^{20}$ produced a major study about this phenomenon by discovering the super-aggregated form of AmB. This new AmB species not only presents an important change in the absorption and circular dichroism spectra of AmB, which is related to its physicochemical state, but also reveals a biological difference when compared to the unheated micelle. ${ }^{21-25}$ Therefore, it can be estimated that the industrial production of a micellar system could be strongly influenced by the manufacturing process, which can modify the surfactant-molecule binding and generate different physicochemical forms.

The results of this study show that, unlike the unheated form, the Brazilian micelle system presents a physicochemical profile quite similar to the European one when heated at $70^{\circ} \mathrm{C}$ for 20 minutes, generating a superaggregated spectroscopic species, which was characterized by a band at $323 \mathrm{~nm}$ on its spectrum. Because this band appeared at the expense of the ones at 327 and $408 \mathrm{~nm}$, the presence of the superaggregated form can be suggested to be a result of the condensation of the aggregated and monomer species of the AmB. This explanation is in complete agreement with the study of Gaboriau et al using a European AmB-DOC (Fungizon $\left.{ }^{\circledR}\right) .{ }^{20}$ Unlike the AmB aggregate species, the dissociation of this super-aggregated form into monomers occurs at lower AmB concentrations. ${ }^{29}$ Therefore, it can be inferred that the AmB super-aggregated species may be considered a reservoir of monomeric AmB species that releases only a limited amount of monomeric $\mathrm{AmB}$ in the aqueous media. As a consequence, the concentration of monomeric AmB might be below its critical aggregation concentration, which is around $10^{-6} \mathrm{M}$; thus, the drug can remain in its monomeric form. This form would be able to bind to the ergosterol of fungal cells, but unable to bind to the cholesterol of the RBCs. ${ }^{10}$

In fact, the variation of patterns of activity against ergosterol-containing fungal cells with respect to toxicity against cholesterol-containing mammalian cells and its 
dependence on the AmB molecular presentation has been reported by several research groups. ${ }^{1,6,26,30}$ It is well-established that the monomeric species of AmB is less effective than the aggregated form in inducing permeability of membranes containing cholesterol, leading to potassium leakage. ${ }^{31}$ The AmB aggregated form was shown to trigger permeability changes in RBC membranes and to induce cytotoxic events. ${ }^{10}$ Such correlation was also maintained in several in vivo studies. ${ }^{32-34}$

The present study reveals that the equilibrium between the different species of $\mathrm{AmB}$ in aqueous media may change and, therefore, its overall activity or toxicity could be changed. The spectral evaluation of both AmB-DOC and AmB-DOC-H clearly demonstrates that the behavior of AmB molecules was modified. For example, the concentration dependence, characteristic of AmB-DOC, disappeared at the concentration of $5 \times 10^{-7} \mathrm{M}$. In fact, at this concentration the AmB super-aggregated band, located at $323 \mathrm{~nm}$, was maintained, whereas the proportion of the monomeric form remained low (Figure 2). This suggests that the AmB molecules were strongly bound to the surfactant molecule and the micelle system had a greater strength than the unheated ones. ${ }^{26}$ Only at the lowest concentration $\left(5 \times 10^{-8} \mathrm{M}\right)$ did both products present a similar spectrum in which the monomeric band had the same shape and similar intensity, reflected by almost the same value of $\varepsilon$.

The reservoir model hypothesis suggested for the superaggregated form of AmB was demonstrated by the in vitro studies. When the target cells were human RBCs, AmBDOC-H was unable to induce hemoglobin leakage over the whole range of doses tested (Figure 4). This demonstrated that the existing aggregated form at $5 \times 10^{-6} \mathrm{M}$ and $5 \times 10^{-5}$ $\mathrm{M}$ was completely different from the AmB-DOC-H product. On the other hand, the activity of both products was quite similar when the cell model was a membrane containing ergosterol, as in the Candida parapisilosis.

The statistical comparison among the different concentrations of AmB for both AmB-DOC and AmB-DOC-H products by the ANOVA test was significant $(P<0.001)$, which confirms that the dilution process induced a decrease in the pattern of activity for all samples. Additionally, Student's $t$-test showed that the toxicity assay against RBCs, represented by the release of $\mathrm{K}^{+}$and hemoglobin, was significantly different from that for AmB-DOC-H, which was much smaller than AmB-DOC $(P<0.05)$.

The probable mechanism behind this pattern of activity could be the high chemical stability of the AmB superaggregates, which are less susceptible to peroxidative process than the aggregated form and, therefore, present less affinity to membranes containing cholesterol than the unheated preparation. ${ }^{8,22}$ Also, ${ }^{8,12}$ this protection against the peroxidation process allows the $\mathrm{AmB}$ super-aggregated form to act as a controlled release of the monomeric $\mathrm{AmB}$ species, which is able to maintain its antifungal activity. ${ }^{10}$ Therefore, as suggested in the literature, a complete resculpting of AmB aggregates probably occurs with the heat-inducing process, and this phenomenon induces changes in the AmB distribution and interaction with various serum fractions. ${ }^{29}$

A mechanistic rationale for the similar efficacy and lower toxicity of AmB-DOC-H, compared to AmB-DOC, as previously proposed, reports that AmB-DOC can be rapidly converted from its aggregated form to a proteinbound monomer in the presence of human serum albumin, whereas AmB-DOC-H demonstrates greater stability by persisting as a stable inactive aggregate. ${ }^{12}$ Moreover, the increased therapeutic index of the AmB-DOC-H may also be the result of greater phagocytosis of the super-aggregates, owing to their larger size $(600 \mathrm{~nm})$, which allows them to be efficiently engulfed by the macrophages and to be transferred to the site of infection in the case of internal cell infections such as leishmaniosis. ${ }^{19}$ On the other hand, it was also shown that the cytotoxicity may be decreased due to a reduction of the interaction of AmB-DOC-H with the kidney cell membranes. ${ }^{35}$

The benefits of the pre-heating process for AmB-DOC $\left(\right.$ Fungizon $^{\circledR}$ ) were also studied in persistently leukopenic mice with severe invasive candidiasis, concluding that higher dosages of AmB-DOC-H, (3.0 versus $0.8 \mathrm{mg} \cdot \mathrm{L}^{-1}$ of body weight), were tolerated, compared to AmB-DOC, resulting in significantly improved therapeutic efficacy. ${ }^{36}$ The cytotoxicity of AmB-DOC-H against pig kidney cells was evaluated by Bartlett et al, who discovered a decrease in the AmB renal cytotoxicity without modifying its antifungal activity. ${ }^{25}$ In the same way, Bau et $\mathrm{al}^{37}$ showed the benefits of the patent-free heated AmB product for use by public-health authorities or a reactive non-governmental organization for treatment of leishmaniasis ${ }^{23}$ and other neglected diseases.

Other important studies have been carried out with the AmB-DOC (Fungizon ${ }^{\circledR}$ ) heated form. For example, a recent work evaluated the fluorescence of $\mathrm{AmB}$ aggregates for the heated and unheated form. The authors concluded that not only the monomer and dimer AmB states but also the aggregate and super-aggregate forms present different spectra. ${ }^{38}$ Therefore, the fluorescence technique could also be used to characterize the different AmB state forms. Rogers et al studied the cytokine and chemokine response elicited by 
AmB-DOC-H in comparison to AmB-DOC in the human monocyte cell line THP- $1 .{ }^{24}$ They concluded that AmB-DOC produced dose-dependent increases in interleukin (IL)-1 $\beta$, IL-1 $\alpha$, tumor necrosis factor- $\alpha$, macrophage inflammatory protein (MIP)- $1 \alpha$, and MIP-1 $\beta$ and that AmB-DOC-H induced cytokine and chemokine production at a lower level than those observed with the corresponding concentrations of AmB-DOC. ${ }^{24}$

\section{Conclusion}

Heat treatment of Fungizon ${ }^{\circledR}$ was shown to modify the aggregation state of the AmB desoxicholate micellar system, generating an AmB super-aggregate species whose appearance is concomitant with the disappearance of both normal aggregates and monomeric $\mathrm{AmB}$ species.

All the results together suggest that the AmB-DOC-H, from a Brazilian industrial company, was less toxic than the unheated form (AmB-DOC) probably due to changes in the AmB aggregation state. It is possible to rebuild micelle systems and generate new entities at a nanoscale level by simple heating. Therefore, we can speculate that this strategy opens the way to creating nanocarriers by changing the manufacturing parameters and process of micelle production.

In short, heat treatment of Fungizon ${ }^{\circledR}$ solutions has been shown to be a simple and inexpensive alternative for treating patients with systemic fungal infections. AmB-DOC-H has been demonstrated to be less toxic to mammalian cells while keeping its activity against fungal cells and protozoan microorganisms. This hypothesis is supported not only by the results presented here, but also by the results found in the literature, which were extensively discussed in this paper.

\section{Acknowledgments}

This work was financially supported by the grant numbers 301979/04-9, 473882/04-3, and 47836/01-7-NV from CNPq and also by UFRN-PPG-PIBIC-CNPq-PPGFar. The authors were grateful to Glenn Hawes, from the University of Georgia, American Language Program, for editing this manuscript.

\section{Disclosure}

The authors report no conflicts of interest in this work.

\section{References}

1. Bolard J. How do the polyene macrolide antibiotics affect the cellular membrane-properties. Biochim Biophys Acta. 1986;864(3-4):257-304.

2. Hartsel S, Bolard J. Amphotericin B: new life for an old drug. Trends Pharmacol Sci. 1996;17(12):445-449.
3. Brajtburg J, Bolard J. Carrier effects on biological activity of amphotericin B. Clinical Microbiology Reviews. 1996;9(4):512-531.

4. Hiemenz JW, Walsh TJ. Lipid formulations of amphotericin B: Recent progress and future directions. Clin Infec Dis. 1996;22: S133-S144.

5. Adams ML, Kwon GS. Relative aggregation state and hemolytic activity of amphotericin B encapsulated by poly(ethylene oxide)-block-poly (N-hexyl-L-aspartamide)-acyl conjugate micelles: effects of acyl chain length. J Control Release. 2003;87(1-3):23-32.

6. Egito EST, Araujo IB, Damasceno B, Price JC. Amphotericin B/ emulsion admixture interactions: an approach concerning the reduction of amphotericin B toxicity. J Pharm Sci. 2002;91(11):2354-2366.

7. Kleinberg M. What is the current and future status of conventional amphotericin B? Int J Antimicrob Agents. 2006;27 Supp1 1: S12-S16.

8. Selvam S, Mishra AK. Disaggregation of amphotericin B by sodium deoxycholate micellar aggregates. J Photochem Photobiol B. 2008; 93(2):66-70.

9. Bolard J, Legrand P, Heitz F, Cybulska B. One-sided action of amphotericin-B on cholesterol-containing membranes is determined by its self-association in the medium. Biochemistry. 1991;30(23): $5707-5715$.

10. Legrand P, Romero EA, Cohen BE, Bolard J. Effects of aggregation and solvent on the toxicity of amphotericin B to human erythrocytes. Antimicrob Agents Chemother. 1992;36(11):2518-2522.

11. Lamy-Freund MT, Ferreira VFN, Faljonialario A, Schreier S. Effect of aggregation on the kinetics of autoxidation of the polyene antibiotic amphotericin-B. J Pharm Sci. 1993;82(2):162-166.

12. Hartsel SC, Bauer E, Kwong EH, Wasan KM. The effect of serum albumin on amphotericin B aggregate structure and activity. Pharm Res. 2001;18(9):1305-1309.

13. Junghanns JU, Buttle I, Muller RH, et al. SolEmuls technology: A way to overcome the drawback of parenteral administration of insoluble drugs. Pharm Dev Technol. 2007;12(5):437-445.

14. Antoniadou A, Dupont B. Lipid formulations of amphotericin B: where are we today? J Mycol Med. 2005;15(4):230-238.

15. Bekersky I, Fielding RM, Buell D, Lawrence I. Lipid-based amphotericin B formulations: from animals to man. Pharm Sci Technol Today. 1999;2(6):230-236.

16. Juliano RL, Grant CWM, Barber KR, Kalp MA. Mechanism of the selective toxicity of amphotericin-B incorporated into liposomes. Mol Pharmacol. 1987;31(1):1-11.

17. Araujo IB, Brito CR, Urbano IA, et al. Similarity between the in vitro activity and toxicity of two different fungizone ${ }^{\mathrm{TM}} /$ lipofundin $^{\mathrm{TM}}$ admixtures. Acta Cir Bras. 2005;20 Suppl 1:257-261.

18. Souza LC, Maranhao RC, Schreier S, Campa A. In-vitro and in-vivo studies of the decrease of amphotericin-B toxicity upon association with a triglyceride-rich emulsion. JAntimicrob Chemother. 1993;32(1): 123-132.

19. Cheron M, Petit C, Bolard J, Gaboriau F. Heat-induced reformulation of amphotericin B-deoxycholate favours drug uptake by the macrophagelike cell line J774. J Antimicrob Chemother. 2003;52(6):904-910.

20. Gaboriau F, Cheron M, Leroy L, Bolard J. Physico-chemical properties of the heat-induced 'super-aggregates' of amphotericin B. Biophys Chem. 1997;66(1):1-12.

21. Gaboriau F, Cheron M, Petit C, Bolard J. Heat-induced superaggregation of amphotericin B reduces its in vitro toxicity: a new way to improve its therapeutic index. Antimicrob Agents Chemother. 1997;41(11): 2345-2351.

22. Hartsel SC, Baas B, Bauer E, et al. Heat-induced superaggregation of Amphotericin B modifies its interaction with serum proteins and lipoproteins and stimulation of TNF-alpha. J Pharm Sci. 2001;90(2): 124-133.

23. Petit C, Yardley V, Gaboriau F, Bolard J, Croft SL. Activity of a heatinduced reformulation of amphotericin B deoxycholate (Fungizone) against Leishmania donovani. Antimicrob Agents Chemother. 1999; 43(2):390-392. 
24. Rogers PD, Barker KS, Herring V, Jacob M. Heat-induced superaggregation of amphotericin $\mathrm{B}$ attenuates its ability to induce cytokine and chemokine production in the human monocytic cell line THP-1. J Antimicrob Chemother. 2003;51(2):405-408.

25. Sivak O, Bartlett K, Wasan KM. Heat-treated Fungizone retains amphotericin $\mathrm{B}$ antifungal activity without renal toxicity in rats infected with Aspergillus fumigatus. Pharm Res. 2004;21(9):1564-1566.

26. Araujo IB, Damasceno BP, de Medeiros TM, Soares LA, do Egito ES. Decrease in Fungizone ${ }^{\mathrm{TM}}$ toxicity induced by the use of Lipofundin ${ }^{\mathrm{TM}}$ as a dilutent: an in vitro study. Curr Drug Deliv. 2005;2(2):199-205.

27. Bartner E, Zinnes H, Moe RA, Kuleska JS. Studies on a new solubilized preparation of amphotericin B. Antibiot Annu. 1957-1958;5:53-58.

28. Ernst C, Dupont G, Rinnert H, Lematre J. Effect of TemperatureChanges on Circular-Dichroism, Absorption-Spectra and LightScattering of Amphotericin-B in Aqueous and Hydro-Alcoholic Solutions. Comptes Rendus Hebdomadaires Des Seances De L Academie Des Sciences Serie B. 1978;286(15):175-178.

29. Baas B, Kindt K, Scott A, Scott J, Mikulecky P, Hartsel SC. Activity and kinetics of dissociation and transfer of amphotericin B from a novel delivery form. AAPS Pharm Sci. 1999;1(3):E10.

30. Caillet J, Berges J, Langlet J. Theoretical study of the self-association of amphotericin B. Biochim Biophys Acta. 1995;1240(2):179-195.

31. Hartsel SC, Benz SK, Ayenew W, Bolard J. $\mathrm{Na}^{+}, \mathrm{K}^{+}$and $\mathrm{Cl}^{-}$Selectivity of the permeability pathways induced through sterol-containing membrane-vesicles by amphotericin-B and other polyene antibiotics. Eur Biophys J. 1994;23(2):125-132.
32. Barwicz J, Christian S, Gruda I. Effects of the aggregation state of amphotericin-B on its toxicity to mice. Antimicrob Agents Chemother. 1992;36(10):2310-2315.

33. Joly V, Farinotti R, Saintjulien L, Cheron M, Carbon C, Yeni P. In vitro renal toxicity and in vivo therapeutic efficacy in experimental murine cryptococcosis of amphotericin-B (Fungizone) associated with Intralipid. Antimicrob Agents Chemother. 1994;38(2):177-183.

34. Swenson CE, Perkins WR, Roberts $P$, et al. In vitro and in vivo antifungal activity of amphotericin B lipid complex: Are phospholipases important? Antimicrob Agents Chemother. 1998;42(4):767-771.

35. Leon C, Taylor R, Bartlett KH, Wasan KM. Effect of heat-treatment and the role of phospholipases on Fungizone ${ }^{\mathbb{R}}$-induced cytotoxicity within human kidney proximal tubular (HK-2) cells and Aspergillus fumigatus. Int J Pharm. 2005;298(1):211-218.

36. van Etten EW, van Vianen W, Roovers P, Frederik P. Mild heating of amphotericin B-desoxycholate: Effects on ultrastructure, in vitro activity and toxicity, and therapeutic efficacy in severe candidiasis in leukopenic mice. Antimicrob Agents Chemother. 2000;44(6):1598-1603.

37. Bau P, Bolard J, Dupouy-Camet J. Heated amphotericin to treat leishmaniasis. Lancet Infect Dis. 2003;3(4):188.

38. Stoodley R, Wasan KA, Bizzotto D. Fluorescence of Amphotericin B-deoxycholate (Fungizone) monomers and aggregates and the effect of heat-treatment. Langmuir. 2007;23(17):8718-8725.
International Journal of Nanomedicine

\section{Publish your work in this journal}

The International Journal of Nanomedicine is an international, peerreviewed journal focusing on the application of nanotechnology in diagnostics, therapeutics, and drug delivery systems throughout the biomedical field. This journal is indexed on PubMed Central, MedLine, CAS, SciSearch ${ }^{\circledR}$, Current Contents ${ }^{\circledR} /$ Clinical Medicine,

\section{Dovepress}

Journal Citation Reports/Science Edition, EMBase, Scopus and the Elsevier Bibliographic databases. The manuscript management system is completely online and includes a very quick and fair peer-review system, which is all easy to use. Visit http://www.dovepress.com/ testimonials.php to read real quotes from published authors. 